\title{
Effect of Predictive Nursing on Preventing from Bone Cement Implantation Syndrome in Hemiarthroplasty
}

\author{
Haiyan Li, Zhiling Ou, Shanghai Ma, Yuyong Liu * \\ The First Affiliated Hospital of Jinan University, Guangzhou, China \\ Email address: \\ docyong@126.com (Yuyong Liu) \\ ${ }^{*}$ Corresponding author \\ To cite this article: \\ Haiyan Li, Zhiling Ou, Shanghai Ma, Yuyong Liu. Effect of Predictive Nursing on Preventing from Bone Cement Implantation Syndrome in \\ Hemiarthroplasty. American Journal of Nursing Science. Vol. 9, No. 1, 2020, pp. 30-34. doi: 10.11648/j.ajns.20200901.15
}

Received: December 27, 2019; Accepted: January 6, 2020; Published: January 17, 2020

\begin{abstract}
This study is to explore the effect of predictive nursing mode on preventing from bone cement implantation syndrome in hemiarthroplasty. 55 patients who were performed with bone cement hemiarthroplasty in our hospital from January 2018 to December 2018 were selected. These patients were divided into conventional group $(\mathrm{n}=25)$ and predictive nursing mode group $(\mathrm{n}=30)$ according to nursing modes. Then the systolic pressure (SBP), diastolic blood pressure (DBP), heart rate (HR) and oxyhemoglobin saturation $\left(\mathrm{SpO}_{2}\right)$ of the two groups of patients were compared 10min before injection of bone cement (T1), at the time of implantation of bone cement (T2), and $10 \mathrm{~min}$ after injection of bone cement respectively (T3). The occurrence situation of respiratory distress, arrhythmia, dizziness, chest distress, panic, nausea, vomiting and shock for the two groups of patients in the surgery were compared. There was no significant difference for the comparison of the data including age, cardiac function, etc. of the patients between the conventional group and predictive nursing mode group. However, SBP and DBP of the intervention group at the time of T1, T2 and T3 were higher than that of the conventional group $(\mathrm{P}<0.05)$. Additionally, $\mathrm{HR}$ and $\mathrm{SpO}_{2}$ of the intervention group at the time of $\mathrm{T} 1, \mathrm{~T} 2$ and $\mathrm{T} 3$ were higher than that of the conventional group $(\mathrm{P}<0.05)$. As for the comparison of the evaluation indexes of bone cement for the two groups of patients in the surgery, the occurrence situation of hypotension, hyoxemia and RDS for the intervention group were apparently lower than that for the conventional group $(\mathrm{P}<0.05)$. As for the comparison of the intraoperative discomfort for the two groups of patients, the occurrence situation of dizziness, chest distress, panic, vomiting, nausea and shock of the intervention group were lower than that of the conventional group. The predictive nursing mode can be used for early intervention so as to relieve and avoid the occurrence of bone cement implantation syndrome in hemiarthroplasty, therefore it is worthy of being promoted for application. Suspected BCIS should be treated with aggressive resuscitation and supportive care. Prevention of BCIS includes identification of high-risk patients, preoperatively optimizing patient risk factors and comorbidities, and good communication with the surgical team.
\end{abstract}

Keywords: Predictive Nursing, Bone Cement Implantation Syndrome, Hemiarthroplasty

\section{Introduction}

As our country gradually steps into aging society, artificial joint replacement has been needed increasingly in the aging society [1]. In light of the increased number of hip fractures in the elderly above 80 years old, hip arthroplasty is an important issue in this population. Bone cement prosthesis which can be used for instant postoperative fixation with reliable effect is suitable for thighbone marrow cavity with bad bone nature, while coming with bone cement toxic reactions (bone cement implantation syndrome, BCIS) [2, 3]. It included acute blood pressure crisis, arrhythmia, cardiac arrest, possibly in the course of injection of bone cement for aged patients with many kinds of internal medical diseases, especially for the patients with cardiopulmonary insufficiency [2]. BCIS may carry up to a 16-fold increase in 30-day postoperative mortality. The etiology and pathophysiology of BCIS are not fully established; however, results of studies and clinical reports are consistent, citing right ventricular failure secondary to increased pulmonary artery pressure as the cause of systemic hypotension and sudden cardiac arrest [3]. The tolerance to anesthesia and surgery can decrease for the aged patients with hypofunction of various organs with growth of age, and the ability of 
regulating the blood circulation system can decrease for most of patients with hypertension, coronary heart disease, furthermore the patients with severe diseases will die [4]. As for most of the aged patients who come with thighbone marrow cavity with severely bad bone nature, bone cement prosthesis are adopted mostly when artificial hemiarthroplasty is performed, which will increase the occurrence of BCIS in the surgery, threatening the patients' life safety $[5,6]$. Therefore, it is of important significance for prevention to perform predictive nursing intervention in the surgery [7]. The predictive nursing mode performed for the patients who received artificial hemiarthroplasty in 2018 has achieved good effect, providing good reference significance for application of predictive nursing mode in orthopedics.

\section{Materials and Method}

\subsection{General Materials}

55 patients who received artificial hemiarthroplasty in our hospital from January 2018 to December 2018 were selected. Inclusion criteria: (1) the patients aged 75 years and more than 75 years; (2) the patients who received laterally artificial hemiarthroplasty with artificial prosthesis which are bone cement prosthesis; (3) the patients who received combined spinal-epidural anesthesia kept waking state in the surgery; (4) the patients who were performed with surgery and anesthesia with the same one physician; (5) the patients signed the informed consent. Exclusion criteria: (1) the patients with diseases in metal system; (2) the patients who failed to be tolerant of the surgery. Out of 55 patients, there were 44 patients with hypertension, 43 patients with coronary heart disease and 34 patients with diabetes; and there were 22 patients with NYHA classification II in preoperative cardiac function, 33 patients with NYHA classification III; and there were 11 patients with preoperative anesthesia ASA classification II, 43 patients with classification III and 1 patient with classification IV. These patients were divided into control group covering 25 patients and intervention group covering 30 patients randomly according to the sequence number of seeing a doctor, including 10 male patients and 15 female patients, aged $85.08 \pm 4.86$ years, in the control group, and 10 male patients and 20 female patients, aged $82.93 \pm 4.56$ years, in the intervention group. There was no significant difference for the comparison of age, cardiac function, etc. between the two groups $(\mathrm{P}>0.05)$, while with comparability for the data of the two groups (Table 1).

Table 1. The result of comparison analysis of the general data for the two groups.

\begin{tabular}{|c|c|c|c|c|c|c|c|c|c|c|c|}
\hline \multirow{2}{*}{ Group } & \multirow{2}{*}{$\mathbf{n}$} & \multicolumn{2}{|c|}{ Sex (n) } & \multirow{2}{*}{ Age (years) } & \multicolumn{4}{|c|}{ ASA degree (n) } & \multicolumn{3}{|c|}{ NYHA degree (n) } \\
\hline & & Male & Female & & $\mathrm{I}$ & II & III & IV & $\mathrm{I}$ & II & III \\
\hline Control & 25 & 10 & 15 & $85.08 \pm 4.86$ & 0 & 5 & 20 & 0 & 0 & 8 & 17 \\
\hline $\mathrm{T} / \mathrm{Z}$ value & & & & 1.687 & \multicolumn{4}{|c|}{-0.236} & \multicolumn{3}{|l|}{-1.321} \\
\hline$P$ value & & & & 0.0974 & \multicolumn{4}{|c|}{0.814} & \multicolumn{3}{|l|}{0.187} \\
\hline
\end{tabular}

\subsection{Methods}

\subsubsection{Control Group}

The conventional nursing intervention was performed. (1) The circulating nurse visited patients one day before the surgery to grasp the patients' disease situation and complications; (2) The effective vein channel was established on the upper limbs before the surgery; (3) The disease situation change was observed closely in the surgery to monitor the patients' vital signs, and blood pressure with sleeve belt was performed every 5 minutes; (4) The patients were conducted with persistent low-concentration oxygen uptake through nasal catheter in the surgery; (5) The patients' upper bodies were covered with cotton quilt for keeping warmth in the surgery; (6) The patients were preventively performed with $10 \mathrm{mg}$ dexamethasone before implantation of bone cement.

\subsubsection{Intervention Group}

The predictive nursing measures was adopted based on the conventional nursing. (1) The circulating nurse of the operating room came to the ward to visit the patients one day before the surgery, and search the patients' disease situation, so as to comprehensively understand the basic situation of the patients, checking whether there was hypovolemia, heart disease or not. The circulating nurse communicated and discussed with anesthetist, surgeon before the surgery, for the purpose of formulating the preventive measures so as to improve the stress capacity of cardio-pulmonary function, improving surgical tolerance. (2) $18 \mathrm{G}$ or $20 \mathrm{G}$ remaining needle was used for puncture on the upper limbs before the surgery, then blood transfusion tube was used and T-junction was connected. The patients who had difficulty in puncture of peripheral vein were performed with puncture of jugular vein or subclavian vein before the surgery, so as to ensure effective vein channel. (3) The vital signs were observed closely. In addition to the conventional anesthesia monitoring, such senior circulation monitoring as invasive arterial pressure monitoring and arterial blood gas analysis were established before the surgery. The proper effective circulation blood volume was maintained before application of bone cement in the surgery according to invasive arterial pressure monitoring. The vasoactive agents and antiallergic drugs including ephedrine, dopamine, epinephrine hydrochloride, dexamethasone or methylprednisolone were prepared. (4) High-concentration oxygen supply for anaesthetic mask was conducted before implantation of bone cement in the surgery to increase oxygen pressure, and enhancing patients' surgical tolerance. (5) The body temperature of the patients were monitored in the surgery, 
and the upper bodies for these patients were covered with inflatable warm-braw heat preservation carpet for keeping warmth. (6) The patients were asked whether there was discomfort when bone cement was used, including dyspnea, chest pain, panic. (7) The thighbones of the patients were washed by normal saline before implantation of bone cement, and then performed with exhaust decompression of marrow cavity by No. 16 silicone tube when bone cement was implanted.

\subsection{Clinical Observation Indexes}

The systolic pressure (SBP), diastolic blood pressure (DBP), heart rate (HR) and oxyhemoglobin saturation $\left(\mathrm{SpO}_{2}\right)$ of the two groups of patients were compared 10min (T1) before injection of bone cement, at the time (T2) of implantation of bone cement, and 10min (T3) after implantation of bone cement respectively; and the patients' discomfort after implantation of bone cement and the occurrence situation of the evaluation indexes of bone cement implantation syndrome, including arrhythmia, dyspnea, dizziness, hyoxemia for the two groups of patients in the surgery were compared.

\subsection{Statistical Treatment}

The data was analyzed with SPSS 19.0 statistical software. The measurement data was expressed by $\mathrm{x} \pm \mathrm{s}$, and conducted with $\mathrm{t}$ test; the data material was expressed by cases (percentage), and conducted with $\chi^{2}$ test. If $\mathrm{P}<0.05$, the difference is of statistical significance.

\section{Results}

\subsection{Comparison of SBP and DBP at T1, T2 and T3 Between the Two Groups}

The systolic blood pressure was $107.08 \pm 21.68 \mathrm{mmHg}$ in the routine group and $128.23 \pm 17.69 \mathrm{mmHg}$ in the intervention group 10 minutes before the implantation of bone cement. However, at the time of bone cement implantation, the systolic blood pressure of the control group and the intervention group were $120.96 \pm 19.64 \mathrm{mmHg}$ and $110.60 \pm 18.90 \mathrm{mmHg}$, respectively. There was no statistical significance between the two groups $(\mathrm{t}=1.989, \mathrm{P}>0.05)$. However, the systolic blood pressure $(126.73 \pm 12.71 \mathrm{mmHg})$ of the intervention group was significantly higher than that of the control group 10 minutes after the implantation of bone cement. There was statistical significance between the two groups $(\mathrm{t}=-4.181, \mathrm{P}<0.001)$. At the same time, the systolic blood pressure $(70.43 \pm 7.00 \mathrm{mmHg})$ of the intervention group was significantly higher than that of the control group $(61.00 \pm 11.05 \mathrm{mmHg}) 10$ minutes after the implantation of bone cement. There was statistical significance between the two groups $(\mathrm{t}=-3.844, \mathrm{P}<0.001)$. The results show that predictive nursing mode can effectively control blood pressure in hemiarthroplasty.

Table 2. Comparison of SBP and DBP in two groups at different time points.

\begin{tabular}{|c|c|c|c|c|c|c|c|}
\hline \multirow{2}{*}{ Group } & \multirow{2}{*}{$\mathbf{n}$} & \multicolumn{3}{|l|}{ SBP (mmHg) } & \multicolumn{3}{|l|}{ DBP (mmHg) } \\
\hline & & T1 & $\mathbf{T 2}$ & T3 & T1 & $\mathbf{T 2}$ & T3 \\
\hline Control & 25 & $107.08 \pm 21.68$ & $120.96 \pm 19.64$ & $107.08 \pm 21.68$ & $72.64 \pm 12.35$ & $70.24 \pm 12.25$ & $61.00 \pm 11.05$ \\
\hline Predictive Nursing & 30 & $128.23 \pm 17.69$ & $110.60 \pm 18.90$ & $126.73 \pm 12.71$ & $72.67 \pm 11.98$ & $61.07 \pm 9.12$ & $70.43 \pm 7.00$ \\
\hline $\mathrm{T}$ value & & -3.986 & 1.989 & -4.181 & -0.08 & 3.179 & -3.844 \\
\hline$P$ value & & 0.000 & 0.052 & 0.000 & 0.994 & 0.002 & 0.000 \\
\hline
\end{tabular}

\subsection{Comparison of $\mathrm{HR}$ and $\mathrm{SpO}_{2}$ in $\mathrm{T} 1, \mathrm{~T} 2$ and $\mathrm{T3}$ Time Points Between the Two Groups}

The heart rate of routine group and intervention group were $84.04 \pm 14.30 \mathrm{mmHg}$ and $81.97 \pm 11.96$ respectively 10 minutes before the implantation of bone cement. However, the systolic blood pressure of the routine group and the intervention group were $79.39 \pm 21.46$ and $79.57 \pm 9.67$ respectively, there was no statistical significance between the two groups $(\mathrm{t}=-0.040, \mathrm{P}>0.05)$. However, the heart rate of the intervention group $(82.03 \pm 9.08)$ was significantly higher than that of the control group $(75 \pm 12.71) 10$ minutes after the implantation of bone cement, which was statistically significant between the two groups $(\mathrm{t}=-2.28, \mathrm{P}<0.05)$. At the same time, the systolic blood pressure of the intervention group $(98.33 \pm 1.79 \%)$ was significantly higher than that of the control group $(93.52 \pm 6.332 \%) 10$ minutes after the implantation of bone cement. There was statistical significance between the two groups $(\mathrm{t}=-3.984, \mathrm{P}<0.001)$. The results show that predictive nursing mode can effectively control heart rate and blood oxygen saturation in hemiarthroplasty.

Table 3. Comparison of $\mathrm{HR}$ and $\mathrm{SpO}_{2}$ in two groups at different time points.

\begin{tabular}{|c|c|c|c|c|c|c|c|}
\hline \multirow{2}{*}{ Group } & \multirow{2}{*}{$\mathbf{n}$} & \multicolumn{3}{|l|}{ HR } & \multicolumn{3}{|l|}{$\mathrm{SpO}_{2}$} \\
\hline & & T1 & T2 & T3 & T1 & T2 & T3 \\
\hline Control & 25 & $84.04 \pm 14.30$ & $79.39 \pm 21.46$ & $75 \pm 12.71$ & $97.16 \pm 2.495$ & $95.92 \pm 3.499$ & $93.52 \pm 6.332$ \\
\hline Predictive Nursing & 30 & $81.97 \pm 11.96$ & $79.57 \pm 9.67$ & $82.03 \pm 9.08$ & $97.50 \pm 2.193$ & $96.87 \pm 2.873$ & $98.33 \pm 1.79$ \\
\hline $\mathrm{T}$ value & & 0.586 & -0.040 & -2.28 & -0.538 & -1.108 & -3.984 \\
\hline$P$ value & & 0.561 & 0.968 & 0.027 & 0.593 & 0.273 & 0.000 \\
\hline
\end{tabular}




\subsection{Comparison of BCIs Evaluation Indexes Between Two Groups}

In the evaluation indexes of bone cement during operation, there was no statistical difference in the occurrence of cardiac arrest, dyspnea and blood pressure drop between the two groups, but the number of patients with arrhythmia in the intervention group was only 3, significantly less than 13 in the conventional group, with statistical significance between the two groups $(\mathrm{P}<0.05)$ (Table 4$)$.

Table 4. Comparison of BCIs evaluation indexes between two groups.

\begin{tabular}{llllll}
\hline Group & Case (n) & Arrhythmia & Cardiac arrest & Dyspnea & 6 \\
\hline Control & 25 & 13 & 1 & 1 & 10 \\
Predictive Nursing & 30 & 3 & 0 & 4 & 30.564 \\
T value & & 9.618 & 47.291 & 1 & 1 \\
$P$ value & & 0.002 & 1 & 1 & 13.255 \\
\hline
\end{tabular}

\subsection{Comparison of Discomfort Symptoms Between Two Groups After Bone Cement Implantation}

Comparing the discomfort of the two groups, we found that in the intervention group, the situation that 2 patients were dizzy, 1 patient was chest tightness, 0 patient was panic, 0 patient was nausea, 0 patient was vomiting, and 0 patient was of shock performance was significantly lower than that in the routine group $(\mathrm{z}=-3.893, \mathrm{P}<0.001)$. See Table 5 .

Table 5. Comparison of discomfort symptoms after bone cement implantation between the two groups.

\begin{tabular}{lllllll}
\hline Group & Case (n) & Dizzy & Chest tightness & Panic & Nausea & Vomiting \\
\hline Control & 25 & 9 & 2 & 1 & 1 & 1 \\
Predictive Nursing & 30 & 2 & 1 & 0 & 0 & 0 \\
Z value & & -3.893 & & & & \\
P value & 0.000 & & & & \\
\hline
\end{tabular}

\section{Discussion}

\subsection{The Significance of Implementing Predictive Nursing Intervention}

Previous study reported that the incidence of cardiac arrest caused by bone cement implant syndrome was $0.6 \%-10 \%$, and the mortality was $0.02-0.5 \%$ [8]. Predictive nursing is to correctly evaluate the risk of complications, implement targeted nursing, change the previous passive nursing mode, minimize the incidence of complications and improve the prognosis [5, 9]. It is of great significance to carry out predictive nursing mode in artificial cemented hemiarthroplasty. According to the complex condition of patients, comprehensive analysis is carried out, and targeted measures are formulated to effectively avoid or reduce the occurrence of cemented bone syndrome [10].

\subsection{Effect Analysis of Predictive Nursing Intervention Model}

In this study, $\mathrm{SBP}, \mathrm{DBP}, \mathrm{HR}$ and $\mathrm{SpO}_{2}$ in the intervention group were higher than those in the conventional group at $\mathrm{T} 1$, $\mathrm{T} 2$ and T3 time points. In the intervention group, the overall situation of the patients was mastered in advance, and preventive measures were worked out jointly with the surgeons and anesthesiologists. As for the elderly patients, the invasive arterial pressure monitoring should be carried out before the bone cement injection [11]. According to the hemodynamic changes of the patients, before the bone cement implantation, the vascular active drugs should be used reasonably to maintain the effective circulation blood volume to avoid the occurrence of hypotension. The bone cement should be implanted into the medullary cavity, with special attention to the fluctuation of blood pressure [12]. Closely observe whether the patient has dyspnea, palpitation, chest pain, nausea and vomiting, dizziness and headache. Once the patient's condition changes, we should timely find out and deal with it. High concentration oxygen is given to the anesthesia mask to improve the oxygen partial pressure. At the same time, it can be corrected by accelerating the infusion speed. As long as the heart function is allowed after anesthesia, vasoactive drugs, such as ephedrine, dopamine and salt, can be used at the same time Epinephrine to avoid a significant drop in blood pressure and oxygen saturation after bone cement implantation [12]. The incidence of hypotension, hypoxemia and respiratory distress in the intervention group was significantly lower than that in the conventional group, suggesting that the intervention group can reduce the hemodynamic fluctuation during the operation and reduce the reaction of bone cement.

Under the influence of various factors, the elderly patients are prone to hypothermia during operation [13]. Once hypothermia occurs, it will slow down the metabolism of anesthetics, affect the myocardial contractility, renal function and coagulation function, make patients have shivering reaction, increase the probability of complications, and affect the rehabilitation of patients $[6,14]$. In the intervention group, the temperature of the patients was monitored during the operation. According to the temperature of the upper body, the patients were given inflatable, adjustable warm air blanket to keep the balance of the internal environment and maintain the function of the heart and other important organs, which played a certain role in preventing the occurrence of 
bone cement implantation syndrome.

The measures to reduce the reaction of bone cement implantation have been reported in literature, including pulp cavity lavage, adequate hemostasis before bone cement implantation, and pulp cavity exhaust decompression [15]. According to the literature, in the intervention group, normal saline was used to wash the femoral marrow cavity before the bone cement was implanted, and No. 16 silicone rubber tube was used to exhaust and decompress the marrow cavity when the bone cement was implanted.

\section{Conclusion}

To sum up, early identification of risk factors of bone cement implantation syndrome can effectively prevent its occurrence. This study uses predictive nursing mode for early intervention, which can reduce and avoid the occurrence of bone cement implantation syndrome, and is worthy of clinical application. Suspected BCIS should be treated with aggressive resuscitation and supportive care. Prevention of BCIS includes identification of high-risk patients, preoperatively optimizing patient risk factors and comorbidities, and good communication with the surgical team.

\section{References}

[1] Fang M, Noiseux N, Linson E, Cram P. The Effect of Advancing Age on Total Joint Replacement Outcomes. Geriatric orthopaedic surgery \& rehabilitation 2015, 6 (3): 173-179.

[2] Schwarzkopf E, Sachdev R, Flynn J, Boddapati V, Padilla RE, Prince DE. Occurrence, risk factors, and outcomes of bone cement implantation syndrome after hemi and total hip arthroplasty in cancer patients. Journal of surgical oncology 2019, 120 (6): 1008-1015.

[3] Hines CB. Understanding Bone Cement Implantation Syndrome. AANA journal 2018, 86 (6): 433-441.

[4] Kwok DHY, Irwin MG. Preoperative assessment of the orthopaedic patient. Anaest Intens Care M 2018, 19 (4): 151-154.
[5] Shi H, Xiao L, Wang Z. Curative effect of artificial femoral head replacement and its effect on hip joint function and complications of senile patients with femoral intertrochanteric fracture. Experimental and therapeutic medicine 2018, 16 (2): 623-628.

[6] Zagra L, Kjaersgaard-Andersen P. Advances in primary and revision total hip replacement. Hip international: the journal of clinical and experimental research on hip pathology and therapy 2014, 24 Suppl 10: S1.

[7] Liu Q, Zhu H. Application of Predictive Nursing Reduces Psychiatric Complications in ICU Patients after Neurosurgery. Iranian journal of public health 2016, 45 (4): 469-473.

[8] Woo R, Minster GJ, Fitzgerald RH, Jr., Mason LD, Lucas DR, Smith FE. The Frank Stinchfield Award. Pulmonary fat embolism in revision hip arthroplasty. Clinical orthopaedics and related research 1995 (319): 41-53.

[9] Vosler PS, Orsini M, Enepekides DJ, Higgins KM. Predicting complications of major head and neck oncological surgery: an evaluation of the ACS NSQIP surgical risk calculator. Journal of otolaryngology - head \& neck surgery $=$ Le Journal d'oto-rhino-laryngologie et de chirurgie cervico-faciale 2018, 47 (1): 21.

[10] Stirton JB, Maier JC, Nandi S. Total hip arthroplasty for the management of hip fracture: A review of the literature. J Orthop 2019, 16 (2): 141-144.

[11] Govil P, Kakar PN, Arora D, Das S, Gupta N, Govil D, Gupta S, Malohtra A. Bone cement implantation syndrome: a report of four cases. Indian journal of anaesthesia 2009, 53 (2): 214-218.

[12] Qi X, Zhang Y, Pan J, Ma L, Wang L, Wang J. Effect of Bone Cement Implantation on Haemodynamics in Elderly Patients and Preventive Measure in Cemented Hemiarthroplasty. BioMed research international 2015, 2015: 568019.

[13] Soleimanha M, Sedighinejad A, Haghighi M, Nabi BN, Mirbolook AR, Mardani-Kivi M. Hemodynamic and Arterial Blood Gas Parameters during Cemented Hip Hemiarthroplasty in Elderly Patients. The archives of bone and joint surgery 2014, 2 (3): 163-167.

[14] Singh V, Bhakta P, Zietak E, Hussain A. Bone cement implantation syndrome: a delayed postoperative presentation. J Clin Anesth 2016, 31: 274-277.

[15] Vaishya R, Chauhan M, Vaish A. Bone cement. Journal of clinical orthopaedics and trauma 2013, 4 (4): 157-163. 